\title{
Analysis and Simulation of Extended Hydrodynamic Models: The Multi-Valley Gunn Oscillator and MESFET Symmetries
}

\author{
GUI-QIANG CHEN ${ }^{\mathrm{a}}$, JOSEPH W. JEROME ${ }^{\mathrm{a}}$ and CHI-WANG SHU ${ }^{\mathrm{b}}$ \\ a Department of Mathematics, Northwestern University, Evanston, IL $60208,{ }^{\mathrm{b}}$ Division of Applied Mathematics, Brown University, \\ Providence, RI 02912
}

\begin{abstract}
We introduce a novel two carrier hydrodynamic model, which incorporates higher dimensional geometric effects into a one dimensional model. We study (1) the GaAs device in the notched oscillator circuit, and, (2) a MESFET channel, and its symmetries. We present new mathematical results for a reduced model.
\end{abstract}

Keywords: Gunn oscillator, Two carrier hydrodynamic model, MESFET, symmery, geometric structure terms

\section{INTRODUCTION}

In previous work, we have demonstrated the robustness of an algorithm (ENO: Essentially Non-Oscillatory) designed for the simulation of the hydrodynamic model for semiconductors over a wide range of parameters. In [8] and [9], $n^{+}-n-n^{+}$diodes in one dimension and MESFETS in two dimensions were simulated. Here we allow multi-species and geometric source terms.

\subsection{Description of the Gunn Oscillator}

The equations describing an RLC tank circuit, connected to a Gunn oscillator, are:

$$
V_{D}(t)=V_{B}-L \frac{d I(t)}{d t}, \quad I(t)=I_{d}(t)+C \frac{d V_{D}(t)}{d t}+\frac{V_{D}(t)}{R},
$$

where $V_{D}(t)$ is the voltage at the device terminal, $V_{B}$ is the bias voltage, $I(t)$ is the current flowing through the battery, and $C$ is the total capacitance, which includes the so-called cold capacitance. $I_{d}(t)$ is the particle current. In [10], a Monte-Carlo simulation of the Boltzmann equation was used to update $I_{d}(t)$. Earlier, a single valley hydrodynamic model was used by [6], whereas here we employ a two-valley hydrodynamic model. The coupling terms and the system have the structure of [1].

\subsection{Basic MESFET Description}

Next we describe a two dimensional MESFET of the size $0.6 \times 0.2 \mu \mathrm{m}^{2}$. The source and the drain each occupies $0.1 \mu \mathrm{m}$ at the upper left and the upper right, respectively, with a gate occupying $0.2 \mu \mathrm{m}$ at the upper middle (Fig. 1, left). The doping is defined by $n_{d}=3 \times 10^{5}{\mu \mathrm{m}^{-3}}^{-3}[0,0.1] \times[0.15,0.2]$ and in

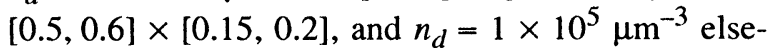



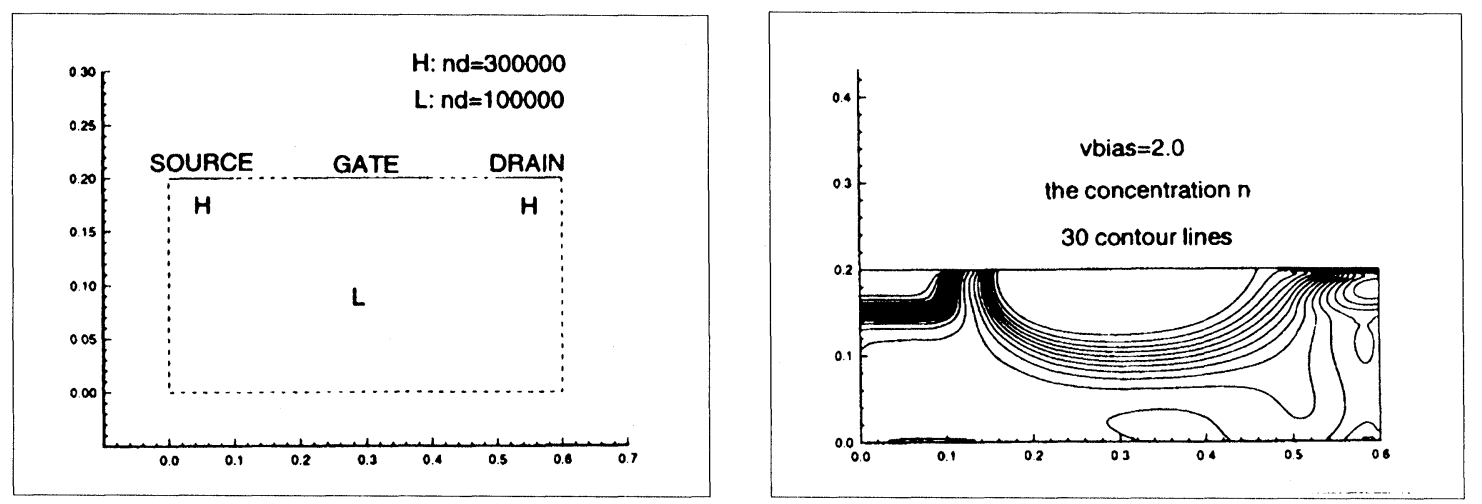

FIGURE 1 Two dimensional MESFET. Left : the geometry and the doping $n_{d}$; right: the contour of the concentration $n$ when vbias $=2.0 \mathrm{~V}$ and vgate $=-0.8 \mathrm{~V}$

where. We apply, at the drain, voltage biases varying up to vbias $=2 \mathrm{~V}$. The gate is a Schottky contact, with negative voltage bias up to vgate $=-0.8 \mathrm{~V}$ and very low concentration value $n=3.8503 \times 10^{-8}{\mu m^{-3}}_{\text {(fol- }}$ lowing Selberherr [12]). The lattice temperature is taken as $T_{0}=300 \mathrm{~K}$.

In Fig. 1, right, we show the contour of the concentration $n$ when vbias $=2 \mathrm{~V}$ and vgate $=-0.8 \mathrm{~V}$. We can see an approximate spherical symmetry around the upper middle point. This serves as a basis for our reduced $1 \mathrm{D}$ model with spherically symmetric forcing terms in the next two sections. The velocity $v$ also shows a similar spherical symmetry. However, temperature $T$ and potential $\Phi$ do not reveal spherical symmetry at this vias.

\section{MATHEMATICAL RESULTS : A WELL POSED REDUCED MODEL}

Consider a reduced model, the compressible, two carrier, Euler-Poisson equations :

$$
\begin{aligned}
& \partial_{t} \rho_{i}+\nabla \cdot \vec{m}_{i}=R_{i}\left(\rho_{1}, \rho_{2}\right), \\
& \partial_{t} \vec{m}_{i}+\nabla \cdot\left(\frac{\vec{m}_{i} \otimes \vec{m}_{i}}{\rho_{i}}\right)+\nabla p\left(\rho_{i}\right) \\
& =\rho_{i} \nabla \phi-\frac{\vec{m}_{i}}{\tau_{i}}+\vec{H}_{i}\left(\rho_{1}, \rho_{2}, E_{1}, E_{2}\right), \\
& \Delta \phi=\rho_{1}+\rho_{2}-n_{d}(\vec{x}), \quad i=1,2, \quad \vec{x} \in \mathbf{R}^{N},
\end{aligned}
$$

where $\rho_{i}(\vec{x}, t), \vec{m}_{i}(\vec{x}, t)$, and $\phi(\vec{x}, t)$ denote the density, the momentum, and the potential of the flows, respectively, and $p\left(\rho_{i}\right)=\rho_{i}^{\gamma / \gamma}, \gamma>1$, is the pressure, $E_{i}=\frac{\rho_{i}^{\gamma-1}}{\gamma(\gamma-1)}+\frac{\left|\vec{m}_{i}\right|^{2}}{2 \rho_{i}^{2}}$ the mechanical energy, $\tau_{i}>0$ is the momentum relaxation time, and $n_{d}(\vec{x})$ is the doping profile. The initial-boundary problem for the system (2.2) with geometrical symmetry is :

$$
\begin{aligned}
& \partial_{t} \rho_{i}+\partial_{x} m_{i}=a(x) m_{i}+R_{i}\left(\rho_{1}, \rho_{2}\right), \\
& \partial_{t} m_{i}+\partial_{x}\left(\frac{m_{i}^{2}}{\rho_{i}}+p\left(\rho_{i}\right)\right) \\
& \quad=a(x) \frac{m_{i}^{2}}{\rho_{i}}+\rho_{i} \phi_{x}-\frac{m_{i}}{\tau_{i}}+H_{i}\left(\rho_{1}, \rho_{2}, E_{1}, E_{2}\right), \\
& \phi_{x x}=a(x) \phi_{x}+\rho_{1}+\rho_{2}-n_{d}(x), \quad i=1,2, \\
& \left.\left(\rho_{i}, m_{i}\right)\right|_{t=0}=\left(\rho_{i 0}(x), m_{i 0}(x)\right), \\
& \left.m_{i}\right|_{x=1}=\left.m_{i}\right|_{x=2}=0, \\
& \left.\quad \phi\right|_{x=1}=\phi_{1}(t) \in \mathbf{L}^{\infty},\left.\quad \phi\right|_{x=2}=\phi_{2}(t) \in \mathbf{L}^{\infty},
\end{aligned}
$$

where the field term $\phi_{x}$ is nonlocal (self-consistent) and $a(x)$ is a $\mathrm{C}^{1}$ function that can be represented by $a(x)=-A^{\prime}(x) / A(x)$. The function $A(x)$ describes the cross-sectional area at $x$ in a variable-area duct such as a nozzle channel, and $A(x)=\frac{2 \pi^{N / 2}}{\Gamma(N / 2)} x^{N-1}$ for spherically symmetric flow in $N$ dimensions, such as 
in the MESFET, for the one carrier case we test in the next section.

The Euler-Possion equations for two carriers with $a(x)=0$ have been studied for some special couplings: The case $R_{i}=H_{i}=0$ in [11] by the Godunov scheme with fractional step techniques and the case $R_{i}=\left(1-\rho_{1} \rho_{2}\right) Q\left(\rho_{1}, \rho_{2}\right), H_{i}=0,0 \leq Q\left(\rho_{1}, \rho_{2}\right) \leq$ $\frac{Q_{0}}{1+\rho_{1}+\rho_{2}}$ in [7] by the viscosity method. The system for one carrier with general $a(x) \in \mathrm{C}^{1}$ is solved in [5].

We develop a new shock capturing numerical scheme and apply this scheme to construct global entropy solutions to the system (2.3-2.4) with nonzero $a(x)$ and general $R_{i}$ and $H_{i}$. More precisely, we consider the following coupling terms $R_{i}\left(\rho_{1}, \rho_{2}\right)$ and $H_{i}\left(\rho_{1}, \rho_{2}, E_{1}, E_{2}\right)$ :

(A1) $R_{i}$ and $H_{i}$ are Lipschitz functions in the variables $\rho_{1} \geq 0, \rho_{2} \geq 0, E_{1}$, and $E_{2}$.

(A2) There exist a constant $C>0$ and a decomposition of $R_{i}: R_{i}\left(\rho_{1}, \rho_{2}\right)=R_{i}^{+}\left(\rho_{1}, \rho_{2}\right)-R_{i}^{-}\left(\rho_{1}, \rho_{2}\right)$ with $R_{i}^{ \pm}\left(\rho_{1}, \rho_{2}\right) \geq 0$ such that, for all $\rho_{1}, \rho_{2}>0$ and $i=1,2$,

$$
\begin{aligned}
& R_{i}\left(\rho_{1}, \rho_{2}\right) \leq C, \quad\left|H_{i}\left(\rho_{1}, \rho_{2}, E_{1}, E_{2}\right)\right| \leq C \rho_{i} ; \\
& \text { When } R_{i}^{+}\left(\rho_{1}, \rho_{2}\right) \geq R_{i}^{-}\left(\rho_{1}, \rho_{2}\right) \\
& \text { and } \rho_{i} \geq(\theta /(\theta+1))^{1 / \theta} \text {, } \\
& R_{i}^{+}\left(\rho_{1}, \rho_{2}\right) \leq C \rho_{i} ; \\
& \text { When } R_{i}^{+}\left(\rho_{1}, \rho_{2}\right)<R_{i}^{-}\left(\rho_{1}, \rho_{2}\right) \text {, } \\
& 0<R_{i}^{-}\left(\rho_{1}, \rho_{2}\right) \leq C \rho_{i} .
\end{aligned}
$$

Theorem 2.1 Let $a(x)$ be $a \mathrm{C}^{1}$ function and $1<\gamma \leq 5 / 3$. Let $R_{i}\left(\rho_{1}, \rho_{2}\right)$ and $H_{i}\left(\rho_{l}, \rho_{2}, E_{1}, E_{2}\right)$ satisfy Assumptions $(A I)-(A 2)$. Then there exists a sequence of approximate solutions $\left(\rho_{i}^{h}(x, t), m_{i}^{h}(x, t)\right)$, for $i=1,2$, converging a.e. to an entropy solution $\left(\rho_{i}(x, t)\right.$, $\left.m_{i}(x, t)\right)$, of (2.3-2.4) such that $0 \leq \rho_{i}(x, t) \leq C(T)<\infty$, $\left|m_{i}(x, t) / \rho_{i}(x, t)\right| \leq C(T)<\infty$, for $0 \leq t \leq T<\infty, x \in \mathbf{R}$, a.e.

Assumptions (A1)-(A2) are in fact quite general. For example, $R_{i}=0, \quad R_{i}=\frac{1-\rho_{1} \rho_{2}}{1+\rho_{1}+\rho_{2}}$, $R_{i}=\frac{(-1)^{i}\left(\rho_{1}-\rho_{2}\right)}{1+\rho_{1}+\rho_{2}}, H_{i}=0$, and $H_{i}=\frac{\rho_{i} E_{i}}{1+E_{1}+E_{2}}$ are in this class. Since $a(x)$ is a nonzero function, the nonlinear resonance between characteristic modes and geometrical source mode occurs at the sonic state, which causes extra difficulties (cf. [3]). Due to the geometrical source terms, we adopt the approach of [3]. Thus, we use the piecewise steady-state solutions, which incorporate such source terms, to replace the piecewise constants from the Riemann solutions as the building blocks. Due to the nonlocal source term, we also incorporate the fractional step procedure into our construction of approximate solutions, with the steady-state solutions as their fundamental building blocks. To obtain a uniform bound for the approximate solutions, we estimate the Riemann invariants involving the nonlocal term with the aid of the conservation of mass and the estimates of the approximate steady-state solutions and Riemann solutions. The $H^{-1}$ compactness of the weak entropy dissipation measures can be achieved as in [2], [3]. These requirements enable us to deduce the strong convergence of the approximate solutions with the aid of a compactness framework (see [2]), proved by DiPerna (1983) for $\gamma=1+\frac{2}{2 s+1}, s \geq 2$ an integer, and by Ding-Chen-Luo (1987) and Chen (1988) for the general case $1<\gamma \leq 5 / 3$.

In [4] we consider the one-dimensional Euler-Poisson equations. It is proved that the relaxation terms prevent the development of shock waves for the smooth initial data with small oscillation, which is not true for large initial data. The nonlinear singular limit of the smooth solution to the drift-diffusion equation is shown when the relaxation times tend to zero.

\section{SIMULATION RESULTS}

We first present simulation results for the Gunn oscillator defined in Section 1.1. Notice that this involves solving a time dependent equation system with strong hyperbolic components, hence upwinding and high order accuracy in space and time are important, justifying the usage of ENO schemes [9]. In Fig. 2, left, we show the time history of the applied voltage $V_{D}(t)$. We can see sustained oscillations of a slow frequency on top of a fast frequency. In Fig. 2 right, we show the 

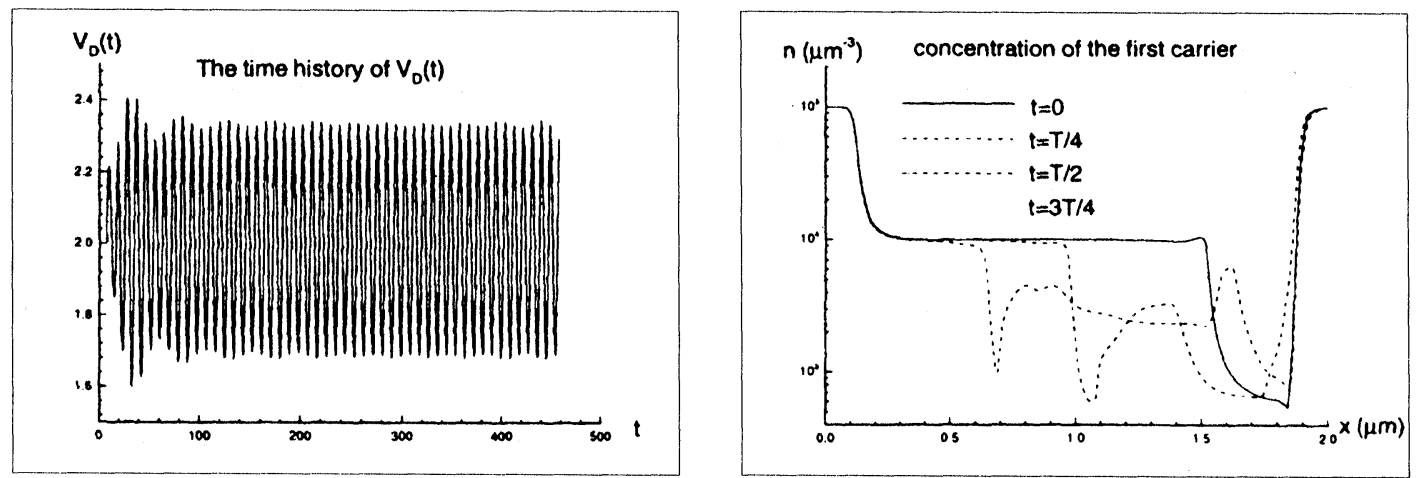

FIGURE 2 Gunn oscillator. Left: the time history of $V_{D}(t)$; right: the concentration $n$ of the first carrier at 4 equally spaced "snaps" over one period of the oscillation

concentration $n$ of the first carrier at 4 equally spaced "snaps" over one period of the oscillation. We can see the movement of the structure clearly in such a period.

Next, we show the result of attempting to use the 1D model with a spherical symmetry assumption, to approximate the 2D MESFET described in Section 1.2. We take our 1D domain to be from $r=0.025$ to $r=0.1$, measured from the top middle point at $(x, y)=(0.3,0.2)$ downwards. The boundary conditions for the concentration $n$, the temperature $T$ and the potential $\phi$ are prescribed, using the values of the 2D simulations; the boundary condition for the velocity is floating (Neumann). In Fig. 3, left, we show the comparison, for the concentration $n$, of the 2D MES-
FET result with the $1 \mathrm{D}$ model assuming spherical symmetry, at vbias $=2.0 \mathrm{~V}$ and vgate $=-0.8 \mathrm{~V}$. We can see a qualitatively correct agreement (the agreement is even better for lower bias). This is good since it means that other quantities (such as $T$ and $\Phi$ ) which are not spherically symmetric have minimal effect on the concentration through the nonlinear coupling of the equations. In Fig. 3, right, we show the same comparison for the temperature $T$. We can see that now the $1 \mathrm{D}$ model disagrees with the $2 \mathrm{D}$ results to a much greater extent, manifesting the fact that $T$ is not spherically symmetric. If $n$ is the only quantity of interest, then the 1D model can be used, saving substantial computing time in the simulation. Otherwise, a better model (perhaps a hybrid one with the non-symmetric
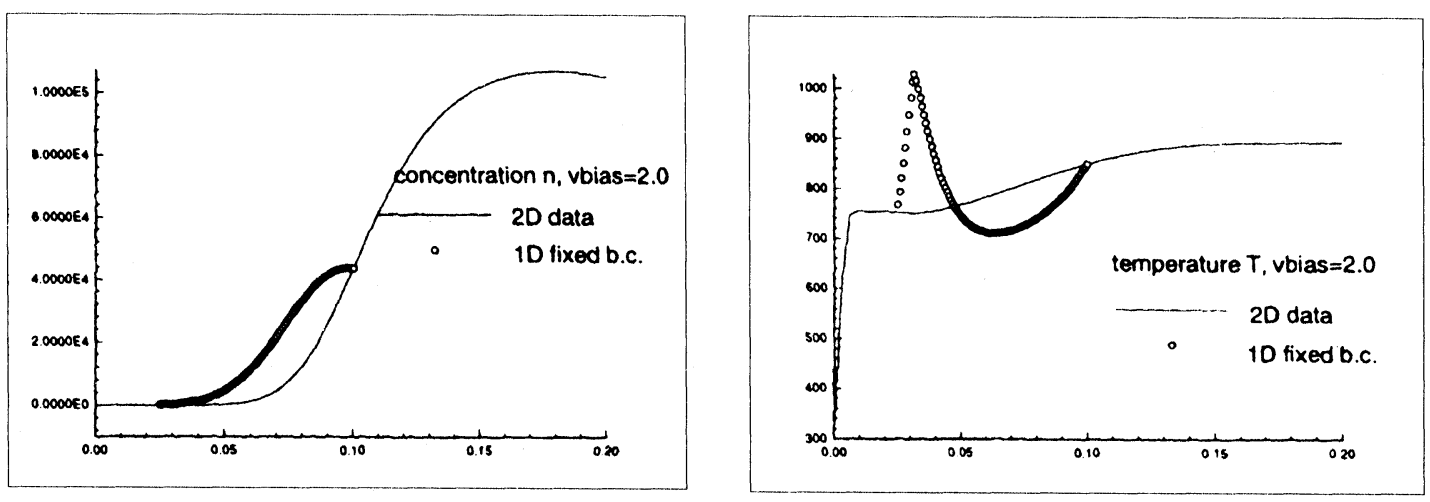

FIGURE 3 The 1D model with spherical symmetry assumption, in comparison with the 2D MESFET results. Left: the concentration $n$; right: the temperature $T$ 
components computed by the 2D model and symmetric quantities computed by the $1 \mathrm{D}$ model) might be useful. This is currently under investigation.

\section{Acknowledgements}

The first author is supported by the Office of Naval Research under grant N00014-91-J-1384, the National Science Foundation under grant DMS-9207080, and by an Alfred P. Sloan Foundation Fellowship. The second author is supported by the National Science Foundation under grant DMS-9424464. The third author is supported by the National Science Foundation under grant ECS-9214488 and the Army Research Office under grant DAAH04-94-G-0205. Computation is supported by the Pittsburgh Supercomputer Center.

\section{References}

[1] K. Blotekjaer. Transport equations for electrons in two-valley semiconductors. IEEE Trans. Electr. Dev., 17:38-47, 1970

[2] G.-Q. Chen. The compensated compactness method and the system of isentropic gas dynamics. MSRI Preprint 00527-9I, Berkeley, 1990

[3] G.-Q. Chen and J. Glimm. Global solutions to the compressible Euler equations with geometrical structure. Commun. Math. Phys. 180: 153-193, 1996

[4] G.-Q. Chen, J.W. Jerome, and B. Zhang. Particle hydrodynamic moment models in biology and microelectronics : singular relaxation limits. Nonlinear Anal. 30:233-244, 1997

[5] G.-Q. Chen and D. Wang. Convergence of shock capturing schemes for the compressible Euler-Poisson equations. Commun. Math. Phys. 179:333-364, 1996

[6] M. Curow and A. Hintz. Numerical simulation of nonstationary electron transport in Gunn devices in a harmonic mode oscillator circuit. IEEE Trans. Electr. Dev. ED-34: 1983-1994, 1987

[7] W. Fang and K. Ito. Weak solutions to a one-dimensional hydrodynamic model of two carrier types for semiconductors. Nonlinear Anal. 28:947-963, 1997

[8] J.W. Jerome and C.-W. Shu. Energy models for one-carrier transport in semiconductor devices. In IMA Volumes in Mathematics and Its Applications, v59, Springer-Verlag, 1994, pp. $185-207$

[9] J.W. Jerome and C.-W. Shu. Transport effects and characteristic modes in the modeling and simulation of submicron devices. IEEE Trans. CADICAS,CAD-14:917-923, 1995

[10] C.H. Lee and U. Ravaioli. Transient Monte-Carlo simulation of heterojunction microwave oscillators. In: K. Hess, J. P. Leburton, and U. Ravaioli (eds.) Computational Electronics. Kluwer Academic Publishers, 1991, pp. 169-172

[11] R. Natalini. The bipolar hydrodynamic model for semiconductors and the drift-diffusion equations. J. Math. Anal. Appl. 198:262-281, 1996

[12] S. Selberherr. Analysis and Simulation of Semiconductor Devices. Springer, 1984

\section{Biographies}

\section{Biographical Sketch of Gui-Qiang Chen:}

Gui-Qiang Chen received the B.S. degree in Mathematics from Fudan University (Shanghai) in 1982 and the $\mathrm{Ph}$. D. degree in Mathematics from Chinese Academy of Sciences (Beijing) in 1987. He was a postdoctoral fellow at the Courant Institute of Mathematical Sciences (New York) in 1987-1989 and an assistant professor of Mathematics at the University of Chicago in 1989-1994, and came to Northwestern University in 1994. He was also a visiting research scientist at the Institute for Mathematics and Its Applications (IMA, Minneapolis) in 1989, at the Mathematical Sciences Research Institute (MSRI, Berkeley) in 1990-1991, and at the Institute for Advanced Study (IAS, Princeton) in 1994. He is now Professor of Mathematics at Northwestern University. His research interests include partial differential equations, nonlinear analysis, numerical analysis, and fluid mechanics. He is currently an Alfred P. Sloan Foundation Fellow (1991-1997) and serves on the editorial board of Zeitschrift für angewandte Mathematik und Physik (ZAMP).

Biographical Sketch of Joseph W. Jerome:

Joseph W. Jerome received the $\mathrm{Ph}$. D. degree in Mathematics from Purdue University in 1966. He was visiting Assistant Professor at the Mathematics Research Center, University of Wisconsin, during 1966-68, and was Assistant Professor at Case Western Reserve University during 1968-70. He joined Northwestern University in 1970, where he has been Professor of Mathematics and Applied Mathematics since 1976. He has held sabbatical positions at Oxford University, England, 1974-75, University of Texas, 1978-79, and Bell Laboratories, Murray Hill, 1982-83. He was visiting scholar at the University of Chicago in 1985. His research interests include applied analysis, numerical analysis, computational electronics, and ion transport in biology. The most recent of his three books, Analysis of Charge Transport, was published by Springer in 1996.

\section{Biographical Sketch of Chi-Wang Shu:}

Chi-Wang Shu received the B.S. degree in Mathematics from the University of Science and Technology of China (USTC) in 1982 and the Ph. D. degree 
in Applied Mathematics from the University of California at Los Angeles (UCLA) in 1986. He was a post doctoral fellow at the Institute for Mathematics and Its Applications (IMA), University of Minnesota, in 1986-1987, and came to Brown University in 1987. $\mathrm{He}$ is now Professor of Applied Mathematics at
Brown University. His research interests include numerical analysis, scientific computing and computational physics. He currently serves on the editorial boards of Mathematics of Computation, SIAM Journal on Numerical Analysis, and Journal of Computational Mathematics. 

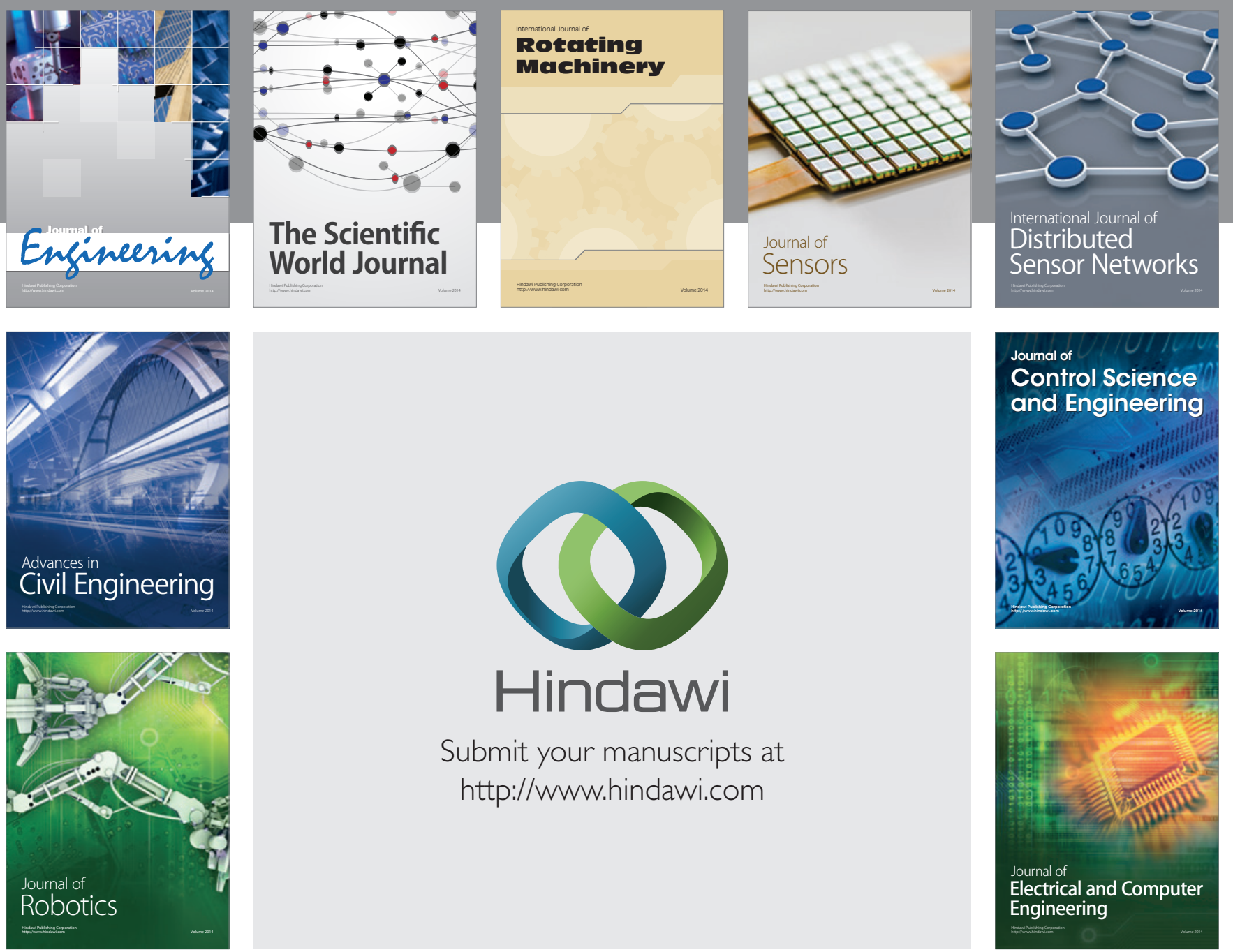

Submit your manuscripts at

http://www.hindawi.com
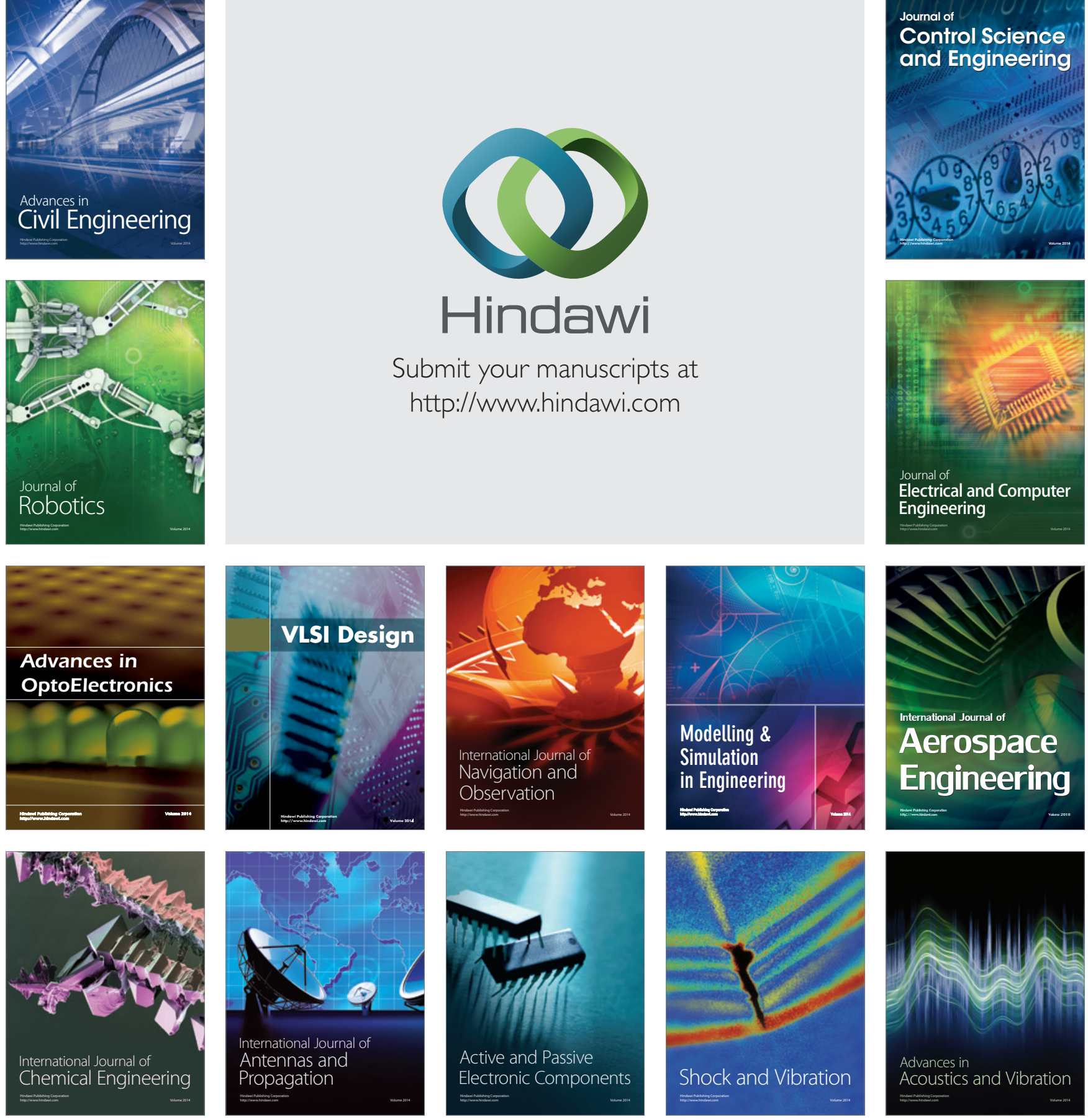